\title{
CASOS E NARRATIVAS - CONTEXTOS E PRETEXTOS PARA A INTEGRAÇÃO DAS TICS NO PROCESSO EDUCATIVO
}

\section{Cases and narratives - contexts and pretexts to integrate ICT's in the educational process}

\author{
Maria Tereza R. Pessoa J. Mendes ${ }^{1}$
}

\section{Resumo}

As complexidades e incertezas que caracterizam a sociedade atual tornam pertinente a valorização da desconstrução e reconstrução de saberes. A formação narrativa representará então, com certeza, um caminho promissor, potencializado pelas TICs, na educação para uma sociedade do conhecimento ou na construção de verdadeiras comunidades aprendentes. Assim, o estudo e a escrita de casos e narrativas pelos processos que envolvem nomeadamente os referentes à própria escrita, em suportes vários e, por outro lado, os que estão implicados nas conversas ou diálogos sucessivos com a experiência ou práticas, rentabilizarão e transformarão os textos locais (poemas, lendas, saberes, sabores ou quaisquer outros recursos tradicionais ou naturais) em contextos virtuais de aprendizagens significativas.

Palavras-chave: Construtivismo; Casos; Histórias; Narrativas digitais.

\section{Abstract}

The complexity and uncertainty that characterise our society highlight the value of deconstructing and reconstructing knowledge. The narrative education supported by ICT's will provide a promising direction to a knowledge society or to the construction of true learning communities. Thus, the study and the writing of cases and narratives through successive dialogues and conversation with local people will transform and improve their texts (e.g. poems, legends, knowledge, tastes or other natural and traditional resources) in virtual leaming contexts as well as in relevant pretexts for those people's learning community.

Keywords: Constructivism; Cases; Stories; Digital narratives.

1 Professora Auxiliar da FPCE-UC. Faculdade de Psicologia e de Ciências da Educação, Universidade de Coimbra, R. do Colégio Novo - Apartado 6153, 3001-802 Coimbra - Portugal. E-Mail: tpessoa@fpce.uc.pt - tpesso@netcabo.pt 


\title{
Introdução
}

\author{
"Ah se não fosse a bruma da manhã \\ nem a velhinha janela onde me vou \\ debruçar para ouvir a voz das coisas \\ eu não era o que sou." \\ Excertos de "Canção duma Sombra", Teixeira de \\ Pascoaes (1907) In Breyner (1998)
}

Este simples poema ou a leitura que, por meio dele, Sophia de Mello Breyner (1998) fez da complexidade da educação não deixa de ser uma for$\mathrm{ma}^{2}$ simples, ou de qualquer modo diferente, de falar de assuntos complexos como os que se referem às finalidades e possibilidades de concretização do ato educativo ou formas de conhecer, crescer e educar.

Serve o mesmo, por um lado, para realçar as possibilidades educativas, estéticas e mesmo éticas, que diferentes formas de falar ou mostrar idéias ou conceitos potencializam. Por outro lado, as palavras, os conteúdos do poema, constituem ajustadas metáforas ao nosso entendimento do que e como se conhece.

\section{Con(texto) 1 - Assim, a voz das coisas ...}

Desde muito cedo, tanto quanto aonde a memória pode levar, que tivemos necessidade de procurar as coisas para as entendermos ou de construir os nossos próprios caminhos, conhecimentos ou compreensões relativas às mesmas, fossem estas a simples planta, o desenvolvimento de uma rã (quantos girinos não vimos!), um eclipse ou o necessário afeto que nos aproximasse de um livro ou do outro. Nunca mais nos iremos esquecer de um desenho da Nossa Senhora que fizemos, com todo o carinho e

2 A importância da forma que se utiliza para falar ou escrever de assuntos complexos foi referenciada e realçada por Wittgenstein na introdução à sua obra Philosophical Investigations (utilizamos a tradução desta obra, de 1987, da responsabilidade da Fundação Calouste Gulbenkian). A estrutura linear subjacente à organização de um livro levou Wittgenstein a preferir representar o seu pensamento sob a forma de um álbum em que cada página representaria uma visita, uma paisagem diferente sobre uma mesma idéia (1987, p.165-166). Le Moigne refere-se também aos constrangimentos que, muitas vezes, uma organização linear de idéias ou artigos impõe e salienta que "provavelmente não existe uma soa ordem», e a desordem é muitas vezes uma ordem diferente" (1999a, p.21). Não poderemos, também, deixar de realçar e utilizar outras formas de conversar sobre a complexidade dos fenômenos que 0 ato educativo pressupõe. Assim, um poema! 
convicção, numa prova da $3^{\mathfrak{a}}$ ou $4^{\underline{a}}$ classe, ainda hoje o consigo desenhar, mas que não foi aceito porque "A Nossa Senhora não era assim!". Segundo a professora, a Nossa Senhora não tinha sardas, não tinha puxinhos, não tinha umas pestanas assim tão grandes, não tinha bochechas e não tinha uma boca assim tão bonita! Tivemos que fazer outra Nossa Senhora, aquela, a da professora, e ainda hoje não percebemos porque é que não gostaram ou aceitaram a nossa Nossa Senhora! Se a professora, naquela altura, nos tivesse escutado, ouvido a nossa voz, se tivesse aceitado como válido o conhecimento que mostramos acerca da imagem da Nossa Senhora, não teria sido, com certeza, necessário ter percorrido tantos caminhos para que hoje pudéssemos afirmar com confiança, a outros, que a Nossa Senhora poderia ser assim, também!

Servem estas reflexões, assentes em percursos pessoais, para mostrar ou estabelecer relações com outras, estas subjacentes a desempenhos profissionais, mas que convergem, ambas, para uma necessária problematização em torno do que é o conhecimento, de como se conhece e da validade desse conhecimento. A idéia da existência de uma realidade, imaterial e essencial, anterior ou independente da observação do sujeito, própria das epistemologias positivistas, parece estar presente na atitude desta, ou muitas outras, professoras. Mesmo que essa realidade seja tida como eternamente velada ou inacessível, como a imagem da Nossa Senhora, permanece inabalável a hipótese ontológica, constitutiva das epistemologias positivistas, que assume a existência da sua essência independentemente da existência ou experiência do sujeito observador. Esta realidade, de alguma forma permanente, torna-se passível de ser conhecida e este conhecimento, legitimado por um critério universal de verdade objetiva, poderá ser, assim, comunicável ou ensinável. A aprendizagem implicará, neste contexto, uma aproximação a um mais desejável ou a uma norma de verdade (norme du vrai) (LE MOIGNE, 1999, p. 41).

A valorização da voz das coisas e das nossas próprias vozes, naturalmente, aproximou-nos da idéia de que o conhecimento implicará, por um lado, um sujeito que conhece e, por outro, que este conhecimento não terá sentido para além deste sujeito. Este tem sido o argumento partilhado pelas diversas epistemologias construtivistas. Parte-se aqui, então, do princípio de que o conhecimento da realidade é construído pelo sujeito a partir da sua própria experiência dessa realidade. Por outro lado, a riqueza desta aprendizagem ou construção dependerá da disponibilidade ou possibilidade do sujeito escutar as vozes, a sua ou a de outros ou a voz das coisas, necessária à valorização dessa experiência. Na medida do valor atribuído à experiência, o conhecimento acontece e torna-se acessível ao sujeito que conhece por meio de um processo de construção de represen- 
tações diversas sobre a mesma em que o sujeito estará, assim, implicado. É nesta relação estreita (inséparable) entre o conhecimento, ou a experiência intencional do sujeito do conhecimento (l'éxpérience intentionnelle du sujet connaissant), e a sua representação, ou a construção titubeante por parte do sujeito (la construction tâtonnant du sujet représentant la connaissance), que se fundamentam ou se definem hoje, no quadro de uma epistemologia construtivista, os conhecimentos passíveis de serem comunicados ou ensinados (LE MOIGNE, 1999, p.70).

Uma outra atitude possível da professora perante a nossa imagem da Nossa Senhora teria sido ouvir a nossa voz, teria sido valorizá-la, como nós e, a partir daí, ter facilitado o desenvolvimento de representações, outras e diversas, num processo, partilhado e aceite ou valorizado, de desenvolvimento ou construção do conhecimento. Desta forma nos situamos em diversos projetos formativos.

É importante, então, que sejam escutadas as vozes para que, a partir delas, se desenvolvam disponibilidades para ouvir a voz dos outros ou a possibilidade de construção do conhecimento ou aprendizagem.

\section{Pre(texto) 1 - Casos, histórias e narrativas digitais}

Narrativas são histórias que descrevem seqüências de ações ou experiências de um certo número de personagens, quer reais e imaginárias ou mesmo virtuais.

A idéia de narrativa não se compreende hoje só no âmbito da criação literária (FERREIRA-ALVES; GONÇALVES, 2001). É um valioso instrumento de construção de identidades, sentidos ou significados individuais ou mesmo das suas circunstâncias e experiências. Trata-se também de uma manifestação cultural fundamental na medida em que valoriza saberes de uma comunidade - mostra como o conhecimento e o sentimento são utilizados, partilhados e social ou colaborativamente negociados numa comunidade. educativo:

É assim, aqui, relevada a importância das narrativas no processo

como metáforas de um novo paradigma do conhecimento (BRUNER, 1990; FERREIRA-ALVES; GONÇALVES, 2001). As narrativas dão expressão à voz das coisas, às nossas próprias vozes, aproximando-nos da idéia de que, como referimos, o conhecimento implica, por um lado, um sujeito que conhece e, por outro, que este conhecimento não terá sentido ou valor para além deste sujeito, argumento partilhado pelas diversas episte- 
mologias construtivistas;

- como instrumentos de educação baseados em Berger (1997). As narrativas são expressões e instrumentos privilegiados de comunicação em Labov (1997) e de construção de conhecimento organizando e integrando ações e acontecimentos do dia-a-dia em episódios significativos (POLKINGHORNE, 1988);

- como forma de pensar e um modo de representar. As pessoas apreendem 0 mundo narrativamente e as pessoas contam 0 mundo narrativamente (RICHARDSON, 1990, apud BERGER, 1997).

Histórias ou narrativas também subjacentes à idéia de casos hoje tão valorizados pelas possibilidades que a sua leitura, análise e mesmo escrita representam na construção do conhecimento - construção esta que se quer ativa, flexível e reflexiva. Casos cuja utilização tem sido largamente fundamentada na construção de comunidades de aprendizagem e, desta forma, na concepção de documentos hipermídia e multimídia (CARVALHO, 1999; FELTOVICH et al., 1989; JACOBSO N; SPIRO, 1991; 1993, 1993a; 1994, 1994a; JACOBSON et al., 1995; MENDES, T. PESSOA, 2001, 2003; MOREIRA, 1996 ; SPIRO et al., 1988; SPIRO et al., 1989; SPIRO; JEHNG, 1990; SPIRO et al., 1991a; SPIRO et al., 1991b).

Casos são "histórias ou narrativas contextualizadas ou situadas num tempo e num espaço que descrevem as situações reais, complexas e multidimensionais que caracterizam a experiência real e, assim, representam conhecimento em utilização e revelam como o personagem principal ou mesmo o escritor, pensa à medida que identifica e resolve problemas" (MENDES, 2001, p.189). Revelam ou implicarão também, por outro lado, o leitor na utilização do conhecimento. Os casos poderão representar, porém, fatias ou cenas dessas mesmas narrativas - os minicasos. Como referem Spiro e Jehng (1990) um caso pode ser uma cena de um filme, um caso médico, um acontecimento histórico, um capítulo de um livro ou mesmo exemplos e episódios relativos a situações concretas e atuais ou virtuais.

\section{Con(texto) 2 - Também, a bruma da manhã ...}

A bruma da manhã, o mistério ou o silêncio das primeiras horas, de qualquer modo a distância necessária ao pensamento e à reflexão. Não basta ter acesso à experiência, seja ela a voz das coisas ou a palavra de outros, para que haja aprendizagem ou construção do conhecimento. É necessário, também, ter tempo para pensar sobre as experiências (RICHERT, 1991). 
O tempo ${ }^{3}$, a necessária pausa e distância relativa às experiências, será uma condição necessária, ou inerente, ao desenvolvimento do pensamento reflexivo compreendido por Dewey (1910), dimensão importante no desenvolvimento ou construção de conhecimento.

O pensamento acontece perante situações ambíguas e problemáticas que apresentam dilemas e alternativas diversas de caminhos ou ações. No entanto, um deslizar suave pelas coisas, subjacente a um sentido mais amplo de pensamento, não implicará reflexão. Esta implicará uma pausa (the suspense of uncertainty), ou o tempo necessário para considerar, ativamente e de forma flexível, outros aspectos importantes na compreensão da situação (DEWEY, 1910, p.11). No pensamento reflexivo esta pausa ganha especial relevo na medida em que representa a possibilidade de procurar outros pontos de vista necessários à construção de problemas.

\section{Pre(texto) 2 - Casos, histórias e narrativas digitais}

Dentre as características e potencialidades das narrativas e dos casos, podemos salientar:

- a sua indiferença factual - isto é, podem ser reais ou virtuais: mais importante do que a veracidade/falsidade ou realidade/possibilidade é a seqüência das frases/imagens/sons e silêncios ou pausas que determinarão os seus enredo e possíveis significados (BRUNER, 1990);

- a sua seqüencialidade - "uma narrativa é composta por uma única seqüência de eventos", segundo Bruner (1990, p.51), que não têm, porém, vida própria. Seqüências diferentes, com momentos de pausa e silêncio diversos, são histórias diferentes;

\footnotetext{
Não podemos deixar de referir o cuidado e reflexão que a idéia de tempo nos tem merecido. Porum lado, a idéia de que o tempo deve ser (bem) utilizado ou não desperdiçado em devaneios ou desvios ou a valonização e relação de uma idéia de tempo com a idéia de um trabalho esforçado e forçado. Como refere Hargreaves, estabeleceu-se, desde o início da Revolução Industrial, uma idéia de tempo vulgarmente expressa como "tempo é, de fato, dinheiro: poupar tempo é também poupar dinheiro" (1998, p.91). Assim foi inventada e desvalorizada a idéia de preguiça, como antinomia necessária e pedagógica para a valorização de um trabalho, assim percebido. No entanto, a preguiça poderá não ser mais do que o tempo necessário, adequado ao ritmo de cada um, para ouvir e considerar outras vozes ou refletir. Nem sempre assim é! O tempo fenomenológico, dimensão do tempo que tem uma duração interiorvariável de indivíduo para indivíduo, é fundamental na construção do conhecimento e, como refere Hargreaves, "o sentido interior de tempo pode estar em desacordo com o tempo medido pelo relógio" (1998, p.111). Acreditamos que sim, que o tempo pessoal tem um papel importante no desenvolvimento de valores e idéias próprias (FULAN; HARGREAVES, 2000, p.84).
} 
- os significados são construídos pelos leitores ou utilizadores em função dos textos, contextos e sucessão de acontecimentos ou 'cenas';

- a sua forma única de criar laços entre o normal e o excepcional (BRUNER, 1990). Pela narrativa é possível dar sentido, negociar significados, explicar ou compreender os desvios ou problemas;

- é uma forma típica de organizar a experiência - os acontecimentos são vistos por meio de um conjunto particular de perspectivas que serão as suas justificações (BRUNER, 1990);

- podem até ensinar, conservar a memória ou alterar o passado (BRUNER, 1990).

Por meio da narrativa "the knower is connected to the known" e a "theory and practice are connected" (BEATTIE, 2000, p.5). O sujeito, enquanto autor ou escritor do caso/narrativa, constrói significado da experiência - as suas vozes - à medida que realiza um esforço "to collect understanding through a form of disciplined recollection", como propôs Sykes (1996) e, enquanto leitor, constrói uma interpretação integrando "his or her experience to the narrative" (SY KES, 1996, p.11). A case ideal implicará, então, uma maior valorização das práticas relativamente às teorias, assim como uma maior valorização da educação narrativa em detrimento do ensino expositivo (SYKES; BIRD, 1992).

Pela narrativa digital e de forma muito particular pelo hipertexto, por outro lado, "é finalmente possível subverter a seqüência espacial do livro, como há muitos os grandes escritores pretendiam, depois de se terem libertado da ordem cronológica dos acontecimentos..." (PEREIRA, 2002, p.49). Outras possibilidades de preencher e definir espaços, outras possibilidades de marcar ritmos e tempos para as nossas próprias histórias. De fato, como refere Pereira "parece indubitável que, sem perder a capacidade de imaginar tudo o que se quiser a partir de uma página, as janelas' do hipertexto abrem outras possibilidades" (2002, p. 49).

\section{Con(texto) 3 - E a velhinha janela...}

A velhinha janela ou outras quaisquer janelas, de qualquer modo outras e diferentes perspectivas ou a necessária flexibilidade no processo de aprendizagem.

Admitindo que a maioria dos sujeitos entra, hoje, para uma instituição educativa por volta dos três anos ou que o fará obrigatoriamente aos seis anos, não podemos deixar de salientar que são muitos anos, dias e horas, 
regra geral até aos vinte e um ou vinte e dois anos que estão sentados numa carteira a ouvir lições, a observar os professores e a analisar situações, de qualquer forma a construir e a desenvolver uma única perspectiva ou modo de olhar a vida, os acontecimentos ou a informação, como alunos que, por certo, com alguns custos de ordem afetiva e cognitiva se transformará.

A ênfase nos programas ou na aquisição de informação favorecerá também nos sujeitos esta mesma atitude ou modo de olhar para as coisas, sejam estas o outro ou outras informações, como alunos. A apreciação ou crítica muitas vezes ouvida ou lida de que a formação é demasiado teórica poderá, porém, mais não significar do que ausência, nestes sujeitos, de outras perspectivas que os tornem capazes de tomarem as dúvidas ou reflexões, de quem escreve e assim conversa sobre as práticas, como suas também. $\mathrm{O}$ problema poderá não estar na teoria, mas na dificuldade dos sujeitos de a tornarem sua ou a ausência de elos, pontes ou situações que exigirão ou implicarão os formandos na consideração ou construção de outros pontos de vista. A flexibilidade, elemento importante do pensamento reflexivo, isto é, na identificação e conceptualização do problema, pela consideração que implica de outros pontos de vista ou contextos é, assim, uma condição ou atitude necessária ao seu desenvolvimento.

\section{Pre(texto) 3 - Casos, histórias e narrativas digitais}

O desenvolvimento de ambientes hipermídia tem-se mostrado promissor como estratégia de formação que permite fazer face às questões levantadas, nomeadamente, no que se refere à integração da teoria e da prática, à interligação de saberes e contextualização das aprendizagens e desenvolvimento de um pensamento reflexivo e flexível.

Para além da facilidade de acesso à informação numa grande variedade de formatos (imagem, som, texto, vídeo) o hipertexto/hipermídia, dada a sua característica de apresentação não-linear da informação, permite ao utilizador uma atitude ativa e de interação dinâmica juntando e subtraindo materiais e informação, cruzando desta forma estas paisagens e criando, de modo flexível, novas ligações entre as suas componentes.

De fato, a não-linearidade, que caracteriza estes ambientes hipertextuais de aprendizagem, é muito importante uma vez que, tal como no mundo real, a aprendizagem não acontece de forma pré-organizada e linear. Desta forma, estes novos ambientes têm condições para fornecer situações semelhantes às que se encontram na realidade: ambientes complexos e com múltiplas representações para exploração e não caminhos predeterminados para atingirem objetivos pré-especificados (MERSETH; LACEY, 1993). Por outro lado, 
como na vida real, nestes ambientes compete ao utilizador e/ou aluno estabelecer as ligações entre as informações segundo o seu próprio ritmo e interesses, tendo assim o aluno um papel mais altivo e de controle da sua própria aprendizagem (AMBROSE, 1991; CONKLIN, 1987; GALL; HANNAFIN, 1994; JONASSEN, 1988; MCALEESE; GREEN, 1990; WOOODHEAD, 1991).

O aspecto da utilização, de forma integrada, de vários tipos de linguagens e mensagens: som, escrita e vídeo é igualmente importante.Hatfield (1996) observa que, de fato, num único documento é possível ilustrar o texto e/ou exemplificar o conceito, isto é, é possível fornecer exemplos alargados de utilização de conhecimentos numa determinada área, promovendo a capacidade de resolução de problemas e/ou tomada de decisões, ou trazer, no nosso caso, para a universidade, exemplos de situações reais, constituindo-se como veículo de comunicação privilegiado ente estes espaços e instituições diferentes e que têm permanecido afastadas (HATFIELD, 1996; MERSETH; LACEY, 1993; NICAISE; BARNES, 1996; SCHLAGAL et al, 1996).

Tem vindo, então, a ser reconhecida a importância da utilização de sistemas de hipertexto e hipermídia em situações educativas apontadas por diversos autores, entre eles podemos citar Collis (1993); Conklin (1987); Duffy e Jonassen (1992); Hatfield (1996); Jonassen (1998); Marchionini (1988); Merseth e Lacey (1993); Nicaise e Barnes (1996); Spiro e Jehng (1990) e, por outro lado, o seu desenvolvimento considerando os princípios da Teoria da Flexibilidade Cognitiva (SPIRO et al., 1988; SPIRO, et al., 1989; SPIRO et al., 1991A, 1991B; SPIRO; JEHNG, 1990).

Rand Spiro e colaboradores diferenciaram situações de aprendizagem em função da fase de aprendizagem e das características conceptuais do domínio de conhecimentos. Assim, grosso modo, podemos distinguir:

- uma fase de aprendizagem introdutória em que é privilegiada a familiaridade com conceitos, testada pela memorização e reconhecimento dos mesmos, fase habitualmente relacionada com domínios de conhecimentos bem-estruturados e simples, com regras e relações hierárquicas facilmente utilizáveis em outras situações;

- e uma fase mais avançada no conhecimento de um determinado domínio e das tarefas a ele associadas, em que se privilegia a capacidade de raciocínio e utilização de conhecimentos em situações as mais diversas possíveis, fase associada a domínios de conhecimentos pouco-estruturados e complexos "onde muitos conceitos são relevantes num caso de aplicação de conhecimentos e os padrões de utilização dos conceitos ou sua combinação varia muito pelos diferentes casos e situações" (SPIRO et al, 1991a, 1991b). 
Um domínio tão complexo e pouco estruturado como é o de aprender a pensar como 'especialista' exigem formas de ensino-aprendizagem que são muitas vezes 'a antítese' das utilizadas em domínios mais simples e bemestruturados, nomeadamente (SPIRO et al., 1988):

- a contextualização das aprendizagens, múltiplas representações dos conhecimentos e a apresentação da informação de forma nãolinear, numa estrutura já não hierárquica, mas em rede.

Spiro et al (1991a; 1991b), assim como Spiro e Jehng, já em 1990, defendem, então, que para aprendizagens com estas características são necessárias novas formas de conceber o processo ensino-aprendizagem e são necessários novos meios para a sua implementação, considerando, então, os ambientes hipermídia como os mais ajustados.

Para além da possibilidade dada aos formandos de construírem 0 seu próprio conhecimento, os defensores de hipermídia salientam a possibilidade dada ao aprendiz, nestes ambientes, de revisitar cenas/situações ou perspectivas rapidamente, facilmente e de forma não-linear (DANIEL, 1996; MCDEVITT,1996; MERSERTH; LACEY, 1993; NIX; SPIRO, 1990; PEREIRA, 1993, 1994; SALOMON,1998; SWAN, 1994). O utilizador, na leitura de uma situação, pode considerar ao mesmo tempo várias perspectivas e variáveis e construir o conhecimento da mesma forma que o conhecimento prático e tácito é construído pelos profissionais. Desta forma, estão a criarem-se as condições necessárias que permitem aos futuros profissionais "poderem pensar, sentir e fazer juízos como especialistas" (MERSERTH; LACEY, 1993, p.292).

As situações práticas apresentadas nestes ambientes, representativas das práticas em toda a sua multidimensionalidade (BLISS; MAZUR,1996; DESBERG; FISHER, 1996), serão altamente contextualizadas e, na sua análise e avaliação, serão considerados vários pontos de vista. Fornecem-se assim aos formandos os instrumentos que os capacitam a explorar as situações em tempo real e a ganharem novos 'insights' sobre as mesmas (MERSERTH; LACEY, 1993). Os ambientes hipermídia oferecem, pois, a possibilidade de experimentar diversos dilemas pertencentes a contextos, situações de prática reais e virtuais.

Dada a flexibilidade cognitiva adquirida pelos formandos, resultado principal destes ambientes de aprendizagem, segundo Spiro etal (1991a), eles estarão não só mais aptos a mudar a sua forma de pensar (sobretudo deixar de pensar como alunos para pensarem como profissionais) como estarão também mais conscientes da mudança no seu próprio pensamento. 


\section{Con(texto) 4 - Não era o que sou ...}

As perspectivas que se acrescentam pela reflexão, partilhada e orientada, em torno de situações educativas reais como as que aconteceram ao longo de um extenso percurso ou caminho de escolaridade que cada um realizou e realiza, constituirão, com certeza, potencialidades de desenvolvimento importantes para que o formando se consiga situar e ser no desempenho profissional.

\section{Pre(texto) 4 - Casos, histórias e narrativas digitais}

As narrativas integram o campo mais vasto das storytelling (narrativas fictícias), segundo Labov (1997) expressão que já vai tendo algum significado no campo da tecnologia educativa embora, neste âmbito, vá tendo já alguma expressão a utilização de casos (CARVALHO, 1999; MENDES, T. PESSOA, 2001, 2003; MOREIRA, 1996; SPIRO; JEHNG, 1990).

A imagem clássica do "contador de histórias" ou "contadores" é a de alguém que, muitas vezes do nada, cria ou elabora um enredo fantástico que nos fascina, prende a atenção e é emocionalmente recompensador (LABOV, 1997): será o caso dos bardos das tribos celtas representados nas histórias de Asterix. A televisão é também um meio narrativo por excelência. A banda desenhada também se enquadra no âmbito das narrativas embora um único cartão (frame) não o seja. Assim como o não será uma só fotografia, mas já o sejam as fotobiografias. O meio que se utiliza para criar e comunicar a narrativa não são indiferentes e afeta a mensagem (BERGER, 1997, p.15)

Hoje vem sendo relevado o papel das TICs na potencialização do valor pedagógico das narrativas (RYAN, 2001). O hipertexto é perspectivado como uma matriz que contém um número infinito de narrativas e, por isso mesmo, poderá ser concebido como um contador de histórias virtual. A natureza interativa do computador potencializa-o então, em termos pedagógicos, no contexto de uma educação narrativa que por sua vez valida a complexidade do conhecimento e a necessária flexibilidade para o seu desenvolvimento (de si e do outro) (FERREIRA-ALVES; GONÇALVES, 2001; RYAN, 2001). As TICs representam a possibilidade de escrever e ler integrando sons e imagens e texto o que, por outro lado, permitirá construir e 'mostrar' os nossos próprios contos - as autobiografias, lendas, contos ou saberes/sabores locais e, desta forma, ensinar e aprender não simplesmente expondo e consumindo informação, mas, sobretudo, construindo conhecimento, produzindo, valorizando e partilhando identidades e contextos ou textos locais. 


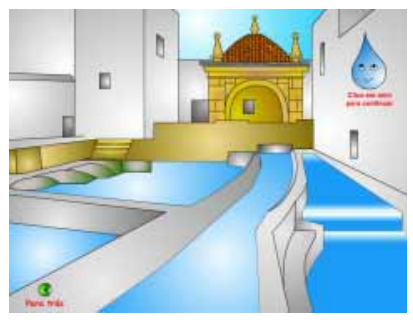

A aplicação multimídia - A Gota de Água construída por Joana Neto no âmbito de um estágio curricular de Ciências da Educação de que somos responsáveis, na Faculdade de Psicologia e de Ciências da Educação da Universidade de Coimbra, conta aspectos ou fatias da história local - Câmara Municipal de Cantanhede - relativa à problemática da água.

Na construção de autênticas comunidades de aprendizagem em que os contextos, e não simplesmente a memorização de textos, têm um papel contributivo inovador e fundamental salientamos, então, a importância destas histórias, casos, narrativas locais a reconstruir, a digitalizar e a partilhar com as histórias de outros, todas elas contextos e pretextos para uma aprendizagem reflexiva e colaborativa ou o necessário caminho para o desenvolvimento de uma cultura aprendente, preocupação maior de uma cidade - real ou virtual - do conhecimento.

\section{Referências}

AMBROSE, D.W. The effects of Hypermedia on Learning: A Literature Review. Educational Technology, v.31, n.12, p.51-55, 1991.

BEATTIE, M. Narratives of Professional Learning: Becoming a Teacher and Learning to Teach. Journal of Educational Inquiry, v.1, n. 2, p. 1-23, 2000. BERGER, A.A. Narratives in popular culture, media, and every day life. California: Sage, 1997.

BLISS, T.; MAZUR, J. Creating a shared culture through cases and technology: The faceless landscape of reform. In: COLBERT, J.; DESBERG, P.; TRIMBLE, K. (Eds). The case for education: contemporary approaches for using case methods. Boston: Allyn and Bacon, 1996. p. 15-28.

BREYNER, S. M. Teixeira de Pascoaes. In: CNE Educação: memórias e testemunhos. Lisboa: Gradiva, 1998. p. 47-48.

BRUNER, J. Actos de significado. Lisboa: Edições 70, 1990.

CARVALHO, A. A. Os hipermédia em contexto educativo: aplicação e validação da teoria da flexibilidade cognitiva. Braga: Centro de Estudos de Educação e Psicologia, Universidade do Minho, 1999. 
COLLS, B. Information technology and teacher education: focus on student learning or on teacher change? Journal of Information Technology for Teacher Education, v. 2, n.2, p.115-125, 1993.

CONKLIN, J. Hypertext: an introduction and survey. IEEE Computer. v. 20, p. 17-41, 1987.

DANIEL, P. Helping beginning teachers link theory to practice: an interactive multimedia environment for mathematics and science teacher preparation. Journal of Teacher Education, v. 47, n.3, p.197-204, 1996.

DESBERG, P; FISHER, F. Using Technology in Case Methodology. In: COLBERT, J.; DESBERG, P. TRIMBLE, K. (Eds). The case for education: Contemporary Approaches for using Case Methods. Boston: Allyn and Bacon, 1996. p. 3955.

DEWEY, J. How we think. Boston: D.C. Heath; CO, 1910.

DUFFY, T. M.; JONASSEN, D. H. Constructivism and the technology of instruction: a conversation. Hillsdale, NJ: Lawrence Erlbaum, 1992.

FELTOVICH, P.J.; SPIRO, R.J.; COULSON, R. L. The nature of conceptual understanding in biomedicine: the deep structure of complex ideas and the development of misconceptions. In: EVANS, D.; PATEL, V. (Eds). The Cognitive Sciences in Medicine. Cambridge, MA: MT, Bradford, 1989. p. 113-172.

FERREIRA-ALVES, J.; GONÇALVES, O. Educação Narrativa do Professor. Coimbra: Quarteto, 2001.

FULLAN, M. HARGREAVES, A. A escola como organização aprendente: buscando uma educação de qualidade. 2 ed. Porto Alegre: Artmed, 2000.

GALL, J.; HANNAFIN, M. A framework for the study of hypertext. Instructional Sciencen,v. 22, p. 207-232, 1994.

HARGREAVES, A. Os professores em tempos de mudança: trabalho e cultura dos professores na idade pós moderna. Lisboa: Mc Graw-Hill, 1998.

HATFIELD, M. Using Multimedia in preservice education. Journal of Teacher Education, v. 47, n. 3, p. 223-227, 1996.

JACOBSON, M.; SPIRO, R. Hypertext Learning Environments and Cognitive Flexibility: Characteristics promoting the transfer of Complex knowledge. In: THE INTERNATIONAL CONFERENCE ON THE LEARNING SCIENCES: Proceedings of the 1991 Conference. Evanston, IL: Northwestern University, 1991. p. 240-248. 
. Hypertext learning environments, cognitive flexibility and the transfer of complex knowledge: an empirical investigation. Champaign, IL: University of Illinois, Center for the Study of Reading, 1993. (Technical Report, n. 573).

. Learning and Applying Difficult Science Knowledge: Research into the application of hypermedia leaming environments First Year Report, University of Illinois, 1993a.

. A framework for the contextual analysis of technology - based learning environments. Journal of Computing in Higher Education, v.5, n.2, p. 3-32, 1994.

. Learning and applying difficult science knowledge: research into the application of hypermedia learning environments Second Year Report, University of Illinois, 1994a.

; MAOURI, C.; MISHRA, P.; KOLAR, C. Learning with hypertext learning environments: Theory, Design, and Research. Journal of Educational Multimedia and Hypermedia. v. 4, n. 4, p. 321-364, 1995.

JONASSEN, D. H. Designing Structured Hypertext, and Structuring Acess to Hypertext. Educational Technology. v. 28, n. 11, p. 13-16, 1998.

LABOV, W. Some Further Steps in Narrative Analysis. Journal of Narrative and Life Story, v. 7, p. 395-415, 1997.

LE MOIGNE, J. Les épistémologies constructivistes. 2. ed. Paris: P.U.F., 1999. 1999 a.

. OConstrutivismo. Volume I: Dos Fundamentos. Lisboa: Instituto Piaget,

MARCHIONINI, G. Hypermedia and Learning: Freedom and Chaos. Educational Technology. v. 28, n. 11, p. 8-12, 1988.

MC ALEESE, R; GREEN, C. (Eds). Hypertext : State of the Art. Oxford: Intellect Limited, 1990.

MCDEVITT, M. A Virtual View: Classroom observations at a distance. Journal of Teacher Education, v. 47, n. 3, p. 191-195, 1996.

MENDES, M. Aprender a pensar como professor: contributo da metodologia de casos na promoção da flexibilidade cognitiva. Tese (Doutorado) - Coimbra: Faculdade de Psicologia e de Ciências da Educação, 2001.

; CARDO SO, I.; NETO, J. Narrativas digitais: textos e contextos para a sua utilização em educação. In:CONFERÊNCIA INTERNACIONALDETECNOLOGIAS 
DE INFORMAÇÃO E COMUNICAÇÃO NA EDUCAÇÃO, 3., : SIMPÓSIO INTERNACIONAL EM INFORMÁTICA EDUCATIVA, 5., Braga, 2003. Anais... Braga: Centro de Competência Nónio Século XXI da Universidade do Minho, 2003. (Actas). p. 771-775. CD-ROM.

MERSETH, K; LACEY, C. Weaving stronger fabric: the pedagogical promise of hypermedia and cases methods in teacher education. Journal of Curriculum Studies, v. 25, n. 6, p. 543-551, 1993.

MOREIRA, A. Desenvolvimento da flexibilidade cognitiva dos alunos, futuros, professores: uma experiência em Didáctica do Inglês. Tese (Doutorado) - Universidade de Aveiro, 1996.

NICAISE, M.; BARNES, D. The Union of Technology, Constructivism, and Teacher Education, Journal of Teacher Education. v. 47, n. 3, p. 205-212, 1996.

NIX, D.; SPIRO, R. Cognition, education, multimedia. Hillside, NJ: Lawrence Erlbaum Associates, 1990.

PEREIRA, D. A Tecnologia educativa e a mudança desejável no sistema educativo. Revista Portuguesa de Educação. v. 6, n. 3, 205-212, 1993.

Reforma perspectivada segundo as novas tecnologias. Revista de Educação. v. 4, n. ', p. 153-162, 1994.

PEREIRA, H. Apologia do hipertexto na deriva do texto. Algés: Difel, 2002. POLKINGHOME, D. Narrative knowing and the human sciences. Albany, NY: Sunny, 1988.

RICHERT, A.. Using teacher cases for reflection and enhanced understanding. In: LEBERMAN, A.; MILER, L. Staff developmentfor education in the'90s. New York: Teachers Collage, 1991. p. 113-132.

RYAN, M. Beyond myth and metaphor: the case of narrative in digital media, 2001. Disponível em: http://www.gamestudies.org/0101/ryan/ Acesso em: 8 Maio 2003.

SALOMAN, G. Novel constructivist learning environments and novel technologies: some issues to be concemed with, 1998. Disponível em http:// cybercon98.harvard.edu/wcm/sal-article.html. Acesso em Maio 2003.

SCHLAGAL, B.; TRATHEN, W; BLANTON, W. Structuring telecommunications to create instructional conversations about student teaching. Journal of Teacher Education. v. 47, n. 3, p. 175-183, 1996.

SPIRO, R; COULSON, R.; FELTOVICH, P.; ANDERSON, D. Cognitive Flexibility 
Theory: Advanced Knowledge Acquisition. In: Tenth Annual Conference of the Cognitive Science Society. Proceedings... Hillsdale, NJ: Enlbaum, 1988. p. 498531.

SPIRO, R; FELTOVICH, P; COULSON, R; ANDERSON, D. Multiple analogies for complex concepts: antidotes for analogy: induced misconceptions in advanced knowledge acquisition. In: VOSNIADOU, S.; ORTONY, A. Similarity and Analogical Reasoning. Cambridge: Cambridge University, 1989. p. 498-531.

SPIRO, R.J.; JEHNG, J. Cognitive flexibility and hypertext: theory and technology for the nonlinear and multidimensional traversal of complex subject matter. In: NIX, D.; SPIRO, R. Cognition, education, and multimedia: exploring ideas in high technology. Hillsdale, NJ: Lawrence Erlbaum Associates, 1990. p. 163-205.

; FELTOVICH, P.J.; JACOBSON, M.J.; COULSON, R. L Cognitive flexibility, constructivism, and hypertext: random acess instruction for advanced knowledge acquisition. Educational Technology. v. 31, n. 5, p. 24-33, 1991a.

SPIRO, R.J.; FELTOVICH, P.J.; JACOBSON, M.J.; COULSON, R. L Knowledge representation, content especification, and the development of skill in situationspecific knowledge assembly: some constructivistissues as they relate to cognitive flexibility theory and hypertext. Educational Technology, v. 31, n. 9, p. 22-25, 1991b.

SYKES, G; BIRD, T. Teacher Education and The Case Idea, 1992. Disponível em: http://ncrtl.nsu.edu/http/sreports/sr892.pdf . Acesso em 23 mar. 2001.

SYKES, G. The case for education-contemporary approaches for using case methods. In: COLBERT, J; KIMBERLEY, T.; DESBERG, P. The case for Educationcontemporary approaches for using case methods. Massachusetts: Allyn; Bacon, Massachusetts, 1996. p. 11.

SWAN, K. History, Hypermedia, and Criss-crossed Conceptual Landscapes. Journal of Educational Multimedia and Hypermedia, v. 3, n. 2, p. 120-139, 1994.

WITTGENSTEIN, L Tratado Lógico: filosófico investigações filosóficas. Lisboa: Fundação Calouste Gulbenkian, 1987.

WOOODHEAD, N. Hypertext; Hypermedia: theory and applications addison. Wilmslow: Wesley, 1991. 